\title{
Unicity of Best Mean Approximation by Second Order Splines with Variable Knots
}

\author{
By D. L. Barrow*, C. K. Chui**, P. W. Smith** and J. D. Ward**
}

Abstract. Let $S_{N}^{2}$ denote the nonlinear manifold of second order splines defined on $[0,1]$ having at most $N$ interior knots, counting multiplicities. We consider the question of unicity of best approximations to a function $f$ by elements of $s_{N}^{2}$. Approximation relative to the $L_{2}[0,1]$ norm is treated first, with the results then extended to the best $L_{1}$ and best one-sided $L_{1}$ approximation problems. The conclusions in each case are essentially the same, and can be summarized as follows: a sufficiently smooth function $f$ satisfying $f^{\prime \prime}>0$ has a unique best approximant from $s_{N}^{2}$ provided either $\log f^{\prime \prime}$ is concave, or $N$ is sufficiently large, $N \geqslant N_{0}(f)$; for any $N$, there is a smooth function $f$, with $f^{\prime \prime}>0$, having at least two best approximants. A principal tool in the analysis is the finite dimensional topological degree of a mapping.

1. Introduction. Let $S_{N}^{2}$ denote the nonlinear manifold of functions which are linear combinations of second order $B$-splines with at most $N$ interior knots on $(0,1)$ counting multiplicities. $S_{N}^{2}$ is the $L_{2}[0,1]$ closure of the class of all piecewise linear continuous functions with at most $N+1$ linear segments. In this article we prove some interesting and somewhat surprising approximation properties of $S_{N}^{2}$ in the space $L_{2}[0,1]$. Three main theorems will be stated in this section with the proofs to follow in later sections. These results are announced in [1].

Theorem 1 describes a fairly large class of uniformly convex functions which have, for each positive integer $N$, unique best $L_{2}[0,1]$ approximants from the (nonlinear) spline manifold $S_{N}^{2}$. Theorem 2 states that any sufficiently smooth uniformly convex function eventually, i.e. for all large $N$, has a unique best $L_{2}[0,1]$ approximant from this manifold. This behavior will be called "eventual uniqueness". Theorem 3 indicates the sharpness of these two results.

We emphasize that $S_{N}^{2}$ is not a linear manifold. Hence, arguments regarding existence, uniqueness, and characterization for best approximants are nontrivial. Since it has been shown in [5] that, for every positive integer $N$, any continuous function has at least one best continuous $L_{2}[0,1]$ approximant from $S_{N}^{2}$, we are only concerned with uniqueness and eventual uniqueness of best approximants in this paper.

TheOREM 1. Let $f \in C^{2}[0,1]$ with $f^{\prime \prime}>0$ on $[0,1]$. Suppose that $\log f^{\prime \prime}$ is concave in $(0,1)$. Then for every positive integer $N$, $f$ has a unique best $L_{2}[0,1]$ approximant from $S_{N}^{2}$.

Received September 19, 1977.

AMS (MOS) subject classifications (1970). Primary 41A15; Secondary 41 A50.

* The research of this author was partially supported by the National Science Foundation under Grant No. DC R75-04545.

** The research of these authors was supported by the U. S. Army Research Office under Grant No. DAHC 04-75-Gं-0186. 
Theorem 2. Let $f \in C^{5}[0,1]$ with $f^{\prime \prime}>0$ on $[0,1]$. Then there exists a positive integer $N_{0}$ such that for any integer $N>N_{0}$, f has a unique best $L_{2}[0,1]$ approximant from $S_{N}^{2}$.

THEOREM 3. Let $N$ be any positive integer. There exists a function $f \in$ $C^{\infty}[0,1]$ with $f^{\prime \prime}>0$ on $[0,1]$, such that $f$ has more than one best $L_{2}[0,1]$ approximant from $S_{N}^{2}$.

We mention that strictly convex $C^{2}[0,1]$ functions always have unique best $L_{\infty}[0,1]$ approximants from $S_{N}^{2}$ (cf. [7, p. 188]). Thus, Theorem 3 shows that there is a fundamental difference in the two norms regarding approximation from $S_{N}^{2}$, and in addition suggests that Theorem 1 is in some sense sharp. This seems to be quite interesting since the class of functions considered in Theorem 1 is not commonly associated with approximation theoretic questions. This work can be thought of as a first step in proving unicity theorems for (generalized) monosplines of minimal norm. The interested reader should consult the first two articles in [6] and the references therein. For a nonuniqueness result on monosplines with least $L_{2}$ norm, see Braess [4].

In establishing the above unicity and eventual unicity results, we will derive a very general method which can be used to yield analogous results in other settings. For example, in Section 7 we will consider the best approximation and best one-sided approximation problems in the $L_{1}$ norm.

2. Preliminaries and Notation. Let $\Sigma^{N} \subset R^{N}$ be the open simplex

$$
\left\{\mathbf{t}^{N}=\left(t_{1}, \ldots, t_{N}\right): 0<t_{1}<\cdots<t_{N}<1\right\} .
$$

We will denote by $\mathbf{t}^{N}$ the $N$ interior knots of a second order spline where $\mathbf{t}^{N}$ could be in the closure of $\Sigma^{N}$ provided $t_{i+2}>t_{i}$ for $i=-1, \ldots, N$, where $t_{-1}=t_{0}=0$, $t_{N+1}=t_{N+2}=1$. With this knot sequence, one can form the normalized $B$-splines

$$
N_{i}\left(\mathrm{t}^{N} ; \tau\right)=N_{i, 2}\left(\mathrm{t}^{N} ; \tau\right)=\left(t_{i+2}-t_{i}\right)\left[t_{i}, t_{i+1}, t_{i+2}\right]_{s}(s-\tau)_{+}
$$

for $i=-1, \ldots, N$ (cf. [2]). Let $S_{N}^{2}=S_{N}^{2}[0,1]$ be the nonlinear manifold of spline functions $s(\cdot)=\Sigma_{i=-1}^{N} A_{i} N_{i}\left(\mathrm{t}^{N} ; \cdot\right)$.

We are interested in the problem of uniqueness of best $L_{2}[0,1]$ approximants from $S_{N}^{2}$. Let $f \in L_{2}[0,1]$ and let $P_{N}(f)$ denote the collection of all best approximants to $f$ from $S_{N}^{2}$ in the $L_{2}[0,1]$ norm. Thus, $s \in P_{N}(f)$ if and only if $s \in S_{N}^{2}$ and

$$
\int_{0}^{1}|f-s|^{2}=\inf \left\{\int_{0}^{1}|f-g|^{2}: g \in S_{N}^{2}\right\} \text {. }
$$

It will be convenient at times to denote the dependence of the spline on its knot sequence. In this case we will write $s=s\left(t^{N}\right)$ which will mean that

$$
s(\cdot)=\sum_{i=-1}^{N} A_{i} N_{i}\left(\mathrm{t}^{N} ; \cdot\right)
$$

for some constants $A_{-1}, \ldots, A_{N}$.

3. Preliminary Lemmas. In this section we will state three lemmas which will be necessary in our proof of Theorems 1 and 2. The first lemma yields the variational 
equations which give a necessary condition satisfied by any best $L_{2}[0,1]$ approximant from $S_{N}^{2}$.

LemMa 1. Let $f$ be in $L_{2}[0,1]$ and $s=s\left(t^{N}\right)$ be in $P_{N}(f)$. Then the restriction of $s$ to any of the subintervals $\left(t_{i}, t_{i+1}\right), i=0, \ldots, N$, is a best linear $L_{2}$ approximant to $f$ on this subinterval. That is,

$$
\int_{t_{i}}^{t_{i+1}}(f-s)(t) t^{j} d t=0, \quad j=0,1 ; i=0, \ldots, N .
$$

This lemma can be easily verified in a standard manner by differentiating the error with respect to both the linear parameters (the coefficients) and the nonlinear parameters (the knots).

The second lemma is a known result which gives some information on the placement of the knots of a best $L_{2}$ approximant.

LEMMA 2. Let $f$ be a continuous function on $[0,1]$ such that $f \notin S_{N}^{2}$ and let $s\left(\mathrm{t}^{N}\right) \in P_{N}(f)$. Then $t_{0}<t_{1}<\cdots<t_{N+1}$ and $s^{\prime}\left(t_{i}^{+}\right) \neq s^{\prime}\left(t_{i}^{-}\right)$for $i=1, \ldots, N$. In particular, $s$ is continuous on $[0,1]$ and all the knots of $s$ are active.

The fact that all knots are active was apparently first observed by de Boor [3]. That $s$ is continuous follows from Theorem 2.3 in [5].

Let $l$ be the best linear $L_{2}[\alpha, \beta]$ approximant to $f$. Then it is easy to verify that

$$
l(\alpha)=-6 \int_{0}^{1}\left(\tau-\frac{2}{3}\right) f(\tau(\beta-\alpha)+\alpha) d \tau
$$

and

$$
l(\beta)=6 \int_{0}^{1}\left(\tau-\frac{1}{3}\right) f(\tau(\beta-\alpha)+\alpha) d \tau
$$

For any knot sequence $\mathrm{t}^{N} \in \Sigma^{N}$, let $\Delta t_{i-1}=t_{i}-t_{i-1}, 1 \leqslant i \leqslant N+1$, and consider the function $\mathbf{F}=\mathbf{F}\left(\mathbf{t}^{N}, f\right)=\left(F_{1}, \ldots, F_{N}\right)$, where

$$
F_{i}=F_{i}\left(\mathrm{t}^{N}, f\right)=-\int_{0}^{1}\left[\left(\tau-\frac{1}{3}\right) f\left(\tau \Delta t_{i-1}+t_{i-1}\right)+\left(\tau-\frac{2}{3}\right) f\left(\tau \Delta t_{i}+t_{i}\right)\right] d \tau
$$

Note that $\mathbf{F}$ is defined and continuous on the closure of $\Sigma^{N}$. From the above two lemmas, we know that if $s=s\left(\mathrm{t}^{N}\right)$ is a best $L_{2}[0,1]$ approximant from $S_{N}^{2}$ to a continuous function $f$, then $\mathbf{F}\left(\mathbf{t}^{N}, f\right)=\mathbf{0}$ where $\mathbf{0}=(0, \ldots, 0)$. Also, for any knot sequence $\mathbf{t}^{N}$, the Jacobian matrix $J\left(\mathbf{F}\left(\mathbf{t}^{N}\right)\right) \equiv\left(\alpha_{i, j}\right)(f)$ of $\mathbf{F}$ defined above as a function of $t_{1}, \ldots, t_{N}$ is a tridiagonal matrix; and if $f$ is in $C^{2}[0,1]$, the only nonzero entries (obtained by differentiating (3.2) and then integrating by parts) are

$$
\begin{array}{rlr}
\alpha_{i, i-1}(f)=\frac{\partial F_{i}}{\partial t_{i-1}}= & -\frac{\Delta t_{i-1}}{3} \int_{0}^{1} \tau(1-\tau)^{2} f^{\prime \prime}\left(\tau \Delta t_{i-1}+t_{i-1}\right) d \tau, & 2 \leqslant i \leqslant N \\
\alpha_{i, i}(f)=\frac{\partial F_{i}}{\partial t_{i}}= & -\frac{\Delta t_{i}}{6} \int_{0}^{1}(2 \tau-1)(1-\tau)^{2} f^{\prime \prime}\left(\tau \Delta t_{i}+t_{i}\right) d \tau \\
& +\frac{\Delta t_{i-1}}{6} \int_{0}^{1}(2 \tau-1) \tau^{2} f^{\prime \prime}\left(\tau \Delta t_{i-1}+t_{i-1}\right) d \tau, \quad 1 \leqslant i \leqslant N
\end{array}
$$


and

(3.5) $\alpha_{i, i+1}(f)=\frac{\partial F_{i}}{\partial t_{i+1}}=-\frac{\Delta t_{i}}{3} \int_{0}^{1} \tau^{2}(1-\tau) f^{\prime \prime}\left(\tau \Delta t_{i}+t_{i}\right) d \tau, \quad 1 \leqslant i \leqslant N-1$.

We have the following result.

Lemma 3. Let $f$ be in $C^{2}[0,1]$ having the properties that $f^{\prime \prime}>0$ and $\log \left(f^{\prime \prime}\right)$ is concave (i.e. $f^{\prime \prime \prime} / f^{\prime \prime}$ is nonincreasing a.e.). If $\mathbf{F}\left(\mathbf{t}^{N}\right)=0$, then the determinant of $J\left(\mathbf{F}\left(\mathbf{t}^{N}\right)\right)$ is positive.

Here, and throughout, $\operatorname{det} A$ denotes the determinant of a matrix $A$. To prove this lemma, we first verify that for each $i, i=2, \ldots, N-1$,

$$
\begin{aligned}
\sum_{j=1}^{N} \alpha_{i, j}(f)= & -\frac{\left(\Delta t_{i}\right)^{2}}{6} \int_{0}^{1} \tau(1-\tau)^{2} f^{\prime \prime \prime}\left(\tau \Delta t_{i}+t_{i}\right) d \tau \\
& +\frac{\left(\Delta t_{i-1}\right)^{2}}{6} \int_{0}^{1} \tau^{2}(1-\tau) f^{\prime \prime \prime}\left(\tau \Delta t_{i-1}+t_{i-1}\right) d \tau
\end{aligned}
$$

Indeed, this follows from integrating (3.2) by parts twice, yielding

$$
\begin{aligned}
F_{i}\left(\mathbf{t}^{N}, f\right)= & -\frac{\left(\Delta t_{i}\right)^{2}}{6} \int_{0}^{1} \tau(1-\tau)^{2} f^{\prime \prime}\left(\tau \Delta t_{i}+t_{i}\right) d \tau \\
& +\frac{\left(\Delta t_{i-1}\right)^{2}}{6} \int_{0}^{1} \tau^{2}(1-\tau) f^{\prime \prime}\left(\tau \Delta t_{i-1}+t_{i-1}\right) d \tau
\end{aligned}
$$

If $\mathbf{t}^{N}$ is a solution to $\mathbf{F}\left(\mathbf{t}^{N}, f\right)=0$, then by (3.6) and (3.7), we have, for $i=2, \ldots, N-1$,

$$
\begin{gathered}
\sum_{j=1}^{N} \alpha_{i, j}(f)=-C\left[\int_{0}^{1} \tau^{2}(1-\tau) f^{\prime \prime}\left(\tau \Delta t_{i-1}+t_{i-1}\right) d \tau\right. \\
\cdot \int_{0}^{1} \tau(1-\tau)^{2} f^{\prime \prime \prime}\left(\tau \Delta t_{i}+t_{i}\right) d \tau \\
-\int_{0}^{1} \tau(1-\tau)^{2} f^{\prime \prime}\left(\tau \Delta t_{i}+t_{i}\right) d \tau \\
\left.\cdot \int_{0}^{1} \tau^{2}(1-\tau) f^{\prime \prime \prime}\left(\tau \Delta t_{i-1}+t_{i-1}\right) d \tau\right],
\end{gathered}
$$

where

$$
C=\frac{\left(\Delta t_{i-1}\right)^{2}}{6} / \int_{0}^{1} \tau(1-\tau)^{2} f^{\prime \prime}\left(\tau \Delta t_{i}+t_{i}\right) d \tau .
$$

Multiplying and dividing the right side of (3.8) by

$$
\left(\int_{0}^{1} \tau^{2}(1-\tau) f^{\prime \prime}\left(\tau \Delta t_{i-1}+t_{i-1}\right) d \tau\right)\left(\int_{0}^{1} \tau(1-\tau)^{2} f^{\prime \prime}\left(\tau \Delta t_{i}+t_{i}\right) d \tau\right)
$$

yields

$$
\sum_{j=1}^{N} \alpha_{i j}(f)=P\left[A_{i-1}-A_{i}\right]
$$


where $P>0$,

and

$$
A_{i}=\int_{0}^{1} \tau(1-\tau)^{2} f^{\prime \prime \prime}\left(\tau \Delta t_{i}+t_{i}\right) d \tau / \int_{0}^{1} \tau(1-\tau)^{2} f^{\prime \prime}\left(\tau \Delta t_{i}+t_{i}\right) d \tau
$$

$$
A_{i-1}=\int_{0}^{1} \tau^{2}(1-\tau) f^{\prime \prime \prime}\left(\tau \Delta t_{i-1}+t_{i-1}\right) d \tau / \int_{0}^{1} \tau^{2}(1-\tau) f^{\prime \prime}\left(\tau \Delta t_{i-1}+t_{i-1}\right) d \tau
$$

Noting that $f^{\prime \prime \prime}=\left(f^{\prime \prime \prime} / f^{\prime \prime}\right) f^{\prime \prime}$, we see that $A_{i}$ is a weighted average of $f^{\prime \prime \prime} / f^{\prime \prime}$ over the interval $\left(t_{i}, t_{i+1}\right)$. Since $f^{\prime \prime \prime} / f^{\prime \prime}$ is nonincreasing, we conclude that $\Sigma_{j=1}^{N} \alpha_{i, j}(f) \geqslant 0$ for $i=2, \ldots, N-1$.

For $i=1$ and $N$ we can conclude that $\Sigma_{j=1}^{N} \alpha_{i j}(f)>0$ by simply noting that $\alpha_{1,0}$ and $\alpha_{N, N+1}$ are well defined by the right side of (3.3) and (3.5), respectively, and arguing as above. Since the off diagonal elements $\alpha_{i, i-1}(f)$ and $\alpha_{i, i+1}(f)$ are negative by using (3.3) and (3.5), we see that the matrix $J\left(F\left(t^{N}\right)\right)$ is diagonally dominant with strict inequality in the first and last rows. By a standard modification of Gershgorin's theorem, it follows that all the eigenvalues of $J\left(F\left(t^{N}\right)\right)$ are in the open right half of the complex plane. Furthermore, since the matrix has only real entries, the complex eigenvalues come in conjugate pairs, so that the product of all the eigenvalues of $J\left(F\left(\mathbf{t}^{N}\right)\right)$ is positive, and Lemma 3 is proved.

4. Proof of the Uniqueness Theorem. We are now ready to prove Theorem 1. Set $g(x)=x^{2}$. Hence, if $s=s\left(\mathrm{t}^{N}\right)$ is a best $L_{2}[0,1]$ approximant to $g$ from $S_{N}^{2}$, then the only solution to $\mathbf{F}\left(\mathrm{t}^{N}, g\right)=\mathbf{0}$ satisfies $t_{i}=i /(N+1), i=1, \ldots, N$, as can easily be seen from (3.7). That is, $g(x)=x^{2}$ has a unique best $L_{2}[0,1]$ approximant from $S_{N}^{2}$, and the knots of this approximant are equally spaced on $[0,1]$.

Let $f$ be as in Theorem 1 . We will show that $\mathbf{F}\left(\mathrm{t}^{N}, f\right)=\mathbf{0}$ has exactly one solution among all possible knot sequences $t^{N}$. Recall that the topological degree of the smooth mapping $\mathbf{G}$ from a bounded open set $D$ in $\mathbf{R}^{N}$ into $\mathbf{R}^{N}$, where $\mathbf{G}$ is continuous on the closure of $D$, is given (cf. [8, p. 69]) by

$$
\operatorname{deg}(\mathbf{p}, \mathbf{G}, \boldsymbol{D})=\sum_{\mathbf{G}(\mathbf{x})=\mathbf{p}} \operatorname{sign} \operatorname{det} J(\mathbf{G}(\mathbf{x}))
$$

where the sum is taken over all solutions $\mathbf{x} \in D$ of $\mathbf{G}(\mathbf{x})=\mathbf{p}$, as long as the Jacobian does not vanish at $\mathbf{x}$ and $\mathbf{p} \notin \mathbf{G}(\partial D)$. It is known that the degree is invariant under homotopy provided that the functions in the homotopy do not introduce solutions on the boundary of $D$. Again, let $g(x)=x^{2}$ and set

$$
\mathbf{F}^{\lambda}(\cdot) \equiv \mathbf{F}(\cdot,(1-\lambda) g+\lambda f) \text {. }
$$

Clearly, $\lambda \rightarrow \mathbf{F}^{\lambda}$ is a homotopy. Then by (3.7) it is easy to see that $\mathbf{F}^{\lambda}$ does not vanish on the boundary of $\Sigma^{N}$. Hence, it follows that

$$
\operatorname{deg}\left(\mathbf{0}, \mathbf{F}^{\lambda}, \Sigma^{N}\right)=\operatorname{deg}\left(\mathbf{0}, \mathbf{F}^{\mathbf{0}}, \Sigma^{N}\right)
$$

for each $\lambda, 0 \leqslant \lambda \leqslant 1$. Therefore, from Lemma 3 , by using (4.2), we conclude that the number of solutions of $\mathbf{F}^{\mathbf{1}}\left(\mathbf{t}^{N}\right)=\mathbf{0}$ is $\operatorname{deg}\left(\mathbf{0}, \mathbf{F}^{\mathbf{0}}, \Sigma^{N}\right)$. From the fact that the determinant of $J\left(\mathbf{F}^{0}\left(\mathbf{t}^{N}\right)\right)$ is positive and the fact that $\mathbf{F}\left(\mathbf{t}^{N}, g\right)=\mathbf{0}$ has only one 
solution, we conclude that $\operatorname{deg}\left(\mathbf{0}, \mathbf{F}^{0}, \Sigma^{N}\right)=1$. This completes the proof of the theorem.

5. Proof of the Eventual Uniqueness Theorem. Let $f$ satisfy the hypotheses of Theorem 2. By arguing as in Section 4, we obtain

$$
\operatorname{deg}\left(\mathbf{0}, \mathbf{F}, \Sigma^{N}\right)=1
$$

where $\mathbf{F}\left(\mathbf{t}^{N}\right) \equiv \mathbf{F}\left(\mathbf{t}^{N}, f\right)$ is defined by (3.2). To prove Theorem 2, we must show that

$$
\operatorname{det} J\left(\mathbf{F}\left(\mathbf{t}^{N}\right)\right)>0
$$

whenever $\mathrm{t}^{N}$ solves $\mathrm{F}\left(\mathrm{t}^{N}\right)=0$ and $N$ is sufficiently large. The fact that $\mathrm{t}^{N}$ is then the unique solution follows as in Section 4.

The key to proving (5.2) is the following algebraic result.

Proposition 1. Let $A=\left(a_{i, j}\right)$ be a tridiagonal $N \times N$ real matrix with positive diagonal entries. Then if

$$
a_{n, n-1} a_{n-1, n} \leqslant a_{n, n} a_{n-1, n-1}\left(1+\pi^{2} / 4 N^{2}\right) / 4
$$

for $n=2, \ldots, N$, it follows that

$$
\operatorname{det} A>0 \text {. }
$$

Proof. Let $A^{\prime}=\operatorname{diag}\left(2 / a_{i i}\right) A$, so that $A^{\prime}$ has all 2's on the main diagonal and $\operatorname{det} A^{\prime}>0$ if and only if $\operatorname{det} A>0$. For $n=1,2, \ldots, N$, let $u_{n}$ be the determinant of the upper left $n \times n$ submatrix of $A^{\prime}$. Then an expansion of $u_{n}$ about the $n$th column yields

$$
u_{n}=2 u_{n-1}-\left(\frac{2 a_{n-1, n}}{a_{n-1, n-1}}\right)\left(\frac{2 a_{n, n-1}}{a_{n, n}}\right) u_{n-2},
$$

$n=2, \ldots, N$, where we define $u_{0}=1$ and $u_{1}=2$. This can be written as

$$
u_{n}-2 u_{n-1}+u_{n-2} \equiv \Delta^{2} u_{n-2}=c_{n} u_{n-2} \text {, }
$$

where

$$
c_{n}=1-\frac{4 a_{n-1, n} a_{n, n-1}}{a_{n-1, n-1} a_{n, n}} \geqslant-\frac{\pi^{2}}{4 N^{2}}
$$

by (5.3).

Before showing that each $u_{n}>0, n=1, \ldots, N$, and hence that $\operatorname{det} A>0$, we motivate a key equation ((5.8) below) with the following observation. Suppose that $u$ and $v$ solve the problems

$$
\begin{array}{lll}
u^{\prime \prime}(x)=f(x) u(x), & x \geqslant 0, & u(0)=u^{\prime}(0)=1, \\
v^{\prime \prime}(x)=-\omega^{2} v(x), & x \geqslant 0, & v(0)=v^{\prime}(0)=1 .
\end{array}
$$

Then $w \equiv u-v$ satisfies . 


$$
w^{\prime \prime}(x)=-\omega^{2} w(x)+\left(f(x)+\omega^{2}\right) u(x), \quad w(0)=w^{\prime}(0)=0,
$$

and one obtains

$$
w(x)=\int_{0}^{x} \frac{\sin \omega(x-\xi)}{\omega}\left(f(\xi)+\omega^{2}\right) u(\xi) d \xi, \quad x \geqslant 0 .
$$

We remark that the Green's function $\sin \omega(x-\xi) / \omega$ can be written as $a(x-\xi)$ where $a$ solves the problem $a^{\prime \prime}(x)=-\omega^{2} a(x), a(0)=0, a^{\prime}(0)=1$.

We now return to the proof of Proposition 1. Let $\left\{v_{n}\right\},\left\{a_{n}\right\}, n=0, \ldots, N$, satisfy

$$
\Delta^{2} v_{n-2}=v_{n}-2 v_{n-1}+v_{n-2}=-\omega^{2} v_{n-2}, \quad n \geqslant 2, v_{0}=1, v_{1}=2,
$$

and

$$
\Delta^{2} a_{n-2}=-\omega^{2} a_{n-2}, \quad n \geqslant 2, a_{0}=0, a_{1}=1
$$

Now let $w_{n}=u_{n}-v_{n}$, so that

$$
\Delta^{2} w_{n-2}=-\omega^{2} w_{n-2}+\left(c_{n}+\omega^{2}\right) u_{n-2}, \quad n \geqslant 2, w_{0}=w_{1}=0 .
$$

We claim that the sequence defined by

$$
x_{n}=\sum_{k=0}^{n-1} a_{n-1-k}\left(c_{k+2}+\omega^{2}\right) u_{k}, \quad n \geqslant 1, x_{0}=0
$$

equals $\left\{w_{n}\right\}$. Indeed, $x_{1}=0$ since $a_{0}=0$; and we have only to prove that (5.7) holds. A direct calculation gives, for $n \geqslant 2$,

$$
\begin{aligned}
\Delta^{2} x_{n-2}= & x_{n}-2 x_{n-1}+x_{n-2}=\sum_{k=0}^{n-1} a_{n-1-k}\left(c_{k+2}+\omega^{2}\right) u_{k} \\
& -2 \sum_{k=0}^{n-2} a_{n-2-k}\left(c_{k+2}+\omega^{2}\right) u_{k}+\sum_{k=0}^{n-3} a_{n-3-k}\left(c_{k+2}+\omega^{2}\right) u_{k} \\
= & \left(c_{n}+\omega^{2}\right) u_{n-2}+\sum_{k=2}^{n-1}\left(c_{n-k+1}+\omega^{2}\right) u_{n-k-1} \Delta^{2} a_{k} \\
= & \left(c_{n}+\omega^{2}\right) u_{n-2}+\sum_{j=0}^{n-3}\left(c_{j+2}+\omega^{2}\right) u_{j}\left(-\omega^{2} a_{n-3-j}\right) \\
= & -\omega^{2} x_{n-2}+\left(c_{n}+\omega^{2}\right) u_{n-2},
\end{aligned}
$$

and so the claim that $w_{n}=x_{n}$ is proved.

Solving for $\left\{v_{n}\right\},\left\{a_{n}\right\}$ yields

$$
v_{n}=\operatorname{Re}(1+i \omega)^{n}+\operatorname{Im}(1+i \omega)^{n} / \omega, \text { and } a_{n}=\operatorname{Im}(1+i \omega)^{n} / \omega
$$

Consequently, if $N \omega \leqslant \pi / 2$, we have $v_{n}>0$ for $n=0, \ldots, N$ and $a_{n}>0$ for $n=$ $1, \ldots, N$. Finally, using (5.8) for $w_{n}$, with $\omega$ replaced by $\pi / 2 N$ so that $c_{k+2}+\omega^{2}$ 
$\geqslant 0$, one concludes that $w_{n}<0$ is impossible; and hence,

$$
u_{n}=w_{n}+v_{n}>0, \quad n=1, \ldots, N .
$$

This completes the proof of Proposition 1.

Before we can use this proposition, we will need information about the rate at which the $\Delta t_{i}$ 's tend to zero as $N$ increases. The next lemma shows that a mesh, $\mathrm{t}^{N}$, which solves $\mathbf{F}\left(\mathrm{t}^{N}, f\right)=\mathbf{0}$ is in fact quasi-uniform.

It will be convenient at this point to introduce some notation which will facilitate the application of some of the results in this section to Section 7 . For $\mathrm{t}^{N} \in \Sigma^{N}$, let

$$
h_{i}=\Delta t_{i}=t_{i+1}-t_{i}, \quad i=0, \ldots, N, \quad \text { and } \quad \Delta=\max h_{i}, \quad \delta=\min h_{i} .
$$

We rewrite Eq. (3.7) in the form

$$
F_{i}\left(\mathrm{t}^{N}\right)=h_{i-1}^{2} \int_{0}^{1} w(\tau) g\left(t_{i}-\tau h_{i-1}\right) d \tau-h_{i}^{2} \int_{0}^{1} w(\tau) g\left(t_{i}+\tau h_{i}\right) d \tau
$$

where $w(\tau)=\tau(1-\tau)^{2} / 6$ and $g=f^{\prime \prime}$.

LEMMA 4. There is a constant $M>0$, depending on $f$ but not on $N$, such that if $F\left(\mathrm{t}^{N}\right)=0, \Delta / \delta \leqslant M$.

Proof. Let $0<m=\min g(t)$ and $K=\max \left|g^{\prime}(t)\right|, 0 \leqslant t \leqslant 1$. From (5.9) with $F_{i}=0$, we obtain

$$
h_{i}^{2} g\left(\eta_{i}\right)=h_{i-1}^{2} g\left(\eta_{i-1}\right)=h_{i-1}^{2}\left(g\left(\eta_{i}\right)+\theta_{i}\left(\eta_{i-1}-\eta_{i}\right)\right),
$$

where $t_{i-1}<\eta_{i-1}<t_{i}<\eta_{i}<t_{i+1}$ and $\left|\theta_{i}\right| \leqslant K$. Let $\Delta=h_{J}$ and $\delta=h_{I}$, where we assume without loss of generality that $J>I$. Then

$$
\begin{aligned}
(\Delta / \delta)^{2} & =\prod_{i=I+1}^{J}\left(\frac{h_{i}}{h_{i-1}}\right)^{2}=\prod_{i=I+1}^{J}\left[1+\frac{\theta_{i}}{g\left(\eta_{i}\right)}\left(\eta_{i-1}-\eta_{i}\right)\right] \\
& \leqslant \exp \left(\sum_{i=1}^{N}\left|\frac{\theta_{i}}{g\left(\eta_{i}\right)}\left(h_{i}+h_{i-1}\right)\right|\right) \leqslant \exp (2 K / m) \equiv M^{2} .
\end{aligned}
$$

Corollary. $\Delta \leqslant M / N$ and $\delta \geqslant 1 / M(N+1)$.

Proof. Use $(N+1) \delta \leqslant 1$ and $(N+1) \Delta \geqslant 1$.

In the following, we will use the expression " $O\left(\Delta^{p}\right)$ ", where $p$ is a positive integer. This will mean that there are positive numbers $K$ and $\Delta_{0}$, which will depend on $f$ and $w$ but not on $N$, such that

$$
\left|O\left(\Delta^{p}\right)\right| \leqslant K \Delta^{p} \quad \text { if } \Delta \leqslant \Delta_{0} .
$$

The following lemma, in conjunction with Proposition 1 and Lemma 4, will complete the proof of Theorem 2 .

Lemma 5. Let $\mathbf{t}^{N}$ solve the equation $\mathbf{F}\left(\mathbf{t}^{N}\right)=0$, and let $J\left(F\left(t^{N}\right)\right)=\left(\alpha_{i, j}\right)$. Then for $N$ sufficiently large,

$$
\alpha_{i, i}>0, \quad i=1, \ldots, N, \quad \text { and }
$$




$$
\alpha_{i, i+1} \alpha_{i+1, i}=\alpha_{i, i} \alpha_{i+1, i+1}\left(1+O\left(\Delta^{3}\right)\right) / 4, \quad i=1, \ldots, N-1
$$

Proof. Let $g_{i}$ denote $g\left(t_{i}\right), g_{i}^{\prime}=g^{\prime}\left(t_{i}\right)$, etc. Set $F_{i}=0$ in (5.9), and expand $g$ about $t_{i}$, obtaining

$$
\begin{gathered}
h_{i-1}^{2} \int_{0}^{1} w(\tau)\left(g_{i}-\tau h_{i-1} g_{i}^{\prime}+\tau^{2} h_{i-1}^{2} g_{i}^{\prime \prime} / 2+O\left(\Delta^{3}\right)\right) d \tau \\
\quad=h_{i}^{2} \int_{0}^{1} w(\tau)\left(g_{i}+\tau h_{i} g_{i}^{\prime}+\tau^{2} h_{i}^{2} g_{i}^{\prime \prime} / 2+O\left(\Delta^{3}\right)\right) d \tau
\end{gathered}
$$

Let

$$
A=\int_{0}^{1} w(\tau) d \tau, \quad B=\int_{0}^{1} \tau w(\tau) d \tau \text { and } C=\int_{0}^{1} \tau^{2} w(\tau) d \tau / 2 .
$$

This yields the expressions

$$
\begin{gathered}
h_{i-1}^{2}-h_{i}^{2}=\frac{B g_{i}^{\prime}}{A g_{i}}\left(h_{i}^{3}+h_{i-1}^{3}\right)+O\left(\Delta^{5}\right), \\
h_{i-1}-h_{i}=\frac{B g_{i}^{\prime}}{A g_{i}}\left(h_{i}^{2}-h_{i} h_{i-1}+h_{i-1}^{2}\right)+O\left(\Delta^{4}\right) .
\end{gathered}
$$

If one differentiates (5.9) and then expands $g$ about $t_{i}$, one obtains

$$
\begin{aligned}
\frac{\partial F_{i}}{\partial t_{i-1}}= & h_{i-1}\left(-2 A g_{i}+3 B h_{i-1} g_{i}^{\prime}-4 C h_{i-1}^{2} g_{i}^{\prime \prime}+O\left(\Delta^{3}\right)\right) \\
\frac{\partial F_{i}}{\partial t_{i}}= & \left(h_{i-1}+h_{i}\right) 2 A g_{i}+\left(h_{i-1}^{2}-h_{i}^{2}\right)(A-3 B) g_{i}^{\prime} \\
& +\left(h_{i-1}^{3}+h_{i}^{3}\right)(-B+4 C) g_{i}^{\prime \prime}+O\left(\Delta^{4}\right) \\
\frac{\partial F_{i}}{\partial t_{i+1}}= & h_{i}\left(-2 A g_{i}-3 B h_{i} g_{i}^{\prime}-4 C h_{i}^{2} g_{i}^{\prime \prime}+O\left(\Delta^{3}\right)\right)
\end{aligned}
$$

A straightforward calculation gives

$$
\begin{aligned}
\frac{\alpha_{i, i+1} \alpha_{i+1, i}}{4 h_{i}^{2} A^{2} g_{i} g_{i+1}}=1+h_{i}\left(\frac{3 B}{2 A}\right)\left(\frac{g_{i}^{\prime}}{g_{i}}-\frac{g_{i+1}^{\prime}}{g_{i+1}}\right) \\
+h_{i}^{2}\left[\frac{4 C}{2 A}\left(\frac{g_{i+1}^{\prime \prime}}{g_{i+1}}+\frac{g_{i}^{\prime \prime}}{g_{i}}\right)-\frac{9 B^{2}}{4 A^{2}} \frac{g_{i}^{\prime}}{g_{i}} \frac{g_{i+1}^{\prime}}{g_{i+1}}\right]+O\left(\Delta^{3}\right) \\
=1+h_{i}^{2}\left[\frac{g_{i}^{\prime \prime}}{g_{i}}\left(\frac{-3 B+8 C}{2 A}\right)+\left(\frac{g_{i}^{\prime}}{g_{i}}\right)^{2}\left(\frac{6 A B-9 B^{2}}{4 A^{2}}\right)\right]+O\left(\Delta^{3}\right) .
\end{aligned}
$$

Similarly, letting $k_{i}=\left(h_{i}+h_{i-1}\right) / 2 h_{i}$, and $k_{i+1}=\left(h_{i+1}+h_{i}\right) / 2 h_{i}$, one computes 


$$
\frac{\alpha_{i, i} \alpha_{i+1, i+1}}{16 h_{i}^{2} A^{2} g_{i} g_{i+1}}=k_{i} k_{i+1}
$$

$$
\begin{aligned}
& +k_{i}\left[\frac{\left(h_{i}^{2}-h_{i+1}^{2}\right)}{2 h_{i}}\left(\frac{A-3 B}{2 A}\right) \frac{g_{i+1}^{\prime}}{g_{i+1}}+\frac{\left(h_{i}^{3}+h_{i+1}^{3}\right)}{2 h_{i}}\left(\frac{-B+4 C}{2 A}\right) \frac{g_{i}^{\prime \prime}}{g_{i}}\right] \\
& +k_{i+1}\left[\frac{\left(h_{i-1}^{2}-h_{i}^{2}\right)}{2 h_{i}}\left(\frac{A-3 B}{2 A}\right) \frac{g_{i}^{\prime}}{g_{i}}+\frac{\left(h_{i-1}^{3}+h_{i}^{3}\right)}{2 h_{i}}\left(\frac{-B+4 C}{2 A}\right) \frac{g_{i}^{\prime \prime}}{g_{i}}\right]+O\left(\Delta^{3}\right),
\end{aligned}
$$

where we have used $(5.10 \mathrm{a})$ to combine the term involving $\left(h_{i-1}^{2}-h_{i}^{2}\right)\left(h_{i}^{2}-h_{i+1}^{2}\right)$ with the higher order terms. We now use the Eqs. (5.10) to obtain

$$
k_{i} k_{i+1}=1+h_{i}^{2}\left[-\frac{1 B}{2 A}\left(\frac{g_{i}^{\prime}}{g_{i}}\right)^{\prime}+\frac{3}{4}\left(\frac{B}{A} \frac{g_{i}^{\prime}}{g_{i}}\right)^{2}\right]+O\left(\Delta^{3}\right) .
$$

Finally, if we use (5.10a) to substitute for the term $h_{i}^{2}-h_{i+1}^{2}$ and $h_{i-1}^{2}-h_{i}^{2}$ in (5.12), we arrive at

$$
\begin{aligned}
& \frac{\alpha_{i, i} \alpha_{i+1, i+1}}{16 h_{i}^{2} A^{2} g_{i} g_{i+1}} \\
& \quad=1+h_{i}^{2}\left[\frac{g_{i}^{\prime \prime}}{g_{i}}\left(\frac{-3 B+8 C}{2 A}\right)+\left(\frac{g_{i}^{\prime}}{g_{i}}\right)^{2}\left(\frac{6 A B-9 B^{2}}{4 A^{2}}\right)\right]+O\left(\Delta^{3}\right) .
\end{aligned}
$$

A comparison of (5.13) with (5.11) shows that Lemma 5 is correct, and hence completes the proof of Theorem 2 .

6. Proof of the Nonuniqueness Theorem. We now prove Theorem 3 . Let $N$ be a positive integer. We first define a function $f_{l}=f_{l, N}, l>0$, depending on whether $N$ is even or odd, as follows: Let $N=2 k$ or $2 k-1$. Choose an angle $\theta$ with $0<\theta<$ $\pi /(4(k+1)$ ). If $N=1$ (or $k=1$ ), then the choice of $\theta$ is not necessary.

(i) For $N=2 k$, let $f_{l}$ be a continuous piecewise linear function on $[0, k l+1]$ with $f_{l}(0)=0$ and with slopes equal to $\tan j \theta$ on $((j-1) l, j l), j=1, \ldots, k$, and 1 on $(k l, k l+1]$.

(ii) For $N=2 k-1$, let $f_{l}$ be a continuous piecewise linear function on $[0, k l+1]$ with $f_{l}(0)=0$ and with slopes equal to $\tan (j-1) \theta$ on $((j-1) l, j l), j=$ $1, \ldots, k$, and 1 on $(k l, k l+1]$.

We next extend $f_{l}$ to be an even function on $[-k l-1, k l+1]$, i.e. $f_{l}(-x)=$ $f_{l}(x), x \in[0, k l+1]$. Then $f_{l}$ is convex and belongs to $S_{N+1}^{2}[-k l-1, k l+1]$.

Let $s_{l}^{*} \in S_{N}^{2}[-k l-1, k l+1]$ be defined on $(-k l-1, k l)$ as the restriction of $f_{l}$. Hence, $s_{l}^{*}$ is continuous at $k l$ and actually linear on $[(k-1) l, k l+1]$. Let $\epsilon \equiv$ $\left\|f_{l}-s_{l}^{*}\right\|_{2}>0$; note that $\epsilon$ is independent of $l$. Here, and throughout this proof, the $L_{2}$ norms are taken on the interval $[-k l-1, k l+1]$. Suppose that $f_{l}$ has a unique best $L_{2}$ approximant $s_{l}$ from $S_{N}^{2}[-k l-1, k l+1]$. Then $s_{l}$ must be an even function, and for large $l$ the knots $x_{i}=x_{i}(l), i=1, \ldots, N$, of $s_{l}$ must interlace the knots of $f_{l}$ in the following manner: $-k l<x_{1}<-(k-1) l<x_{2}<\cdots<x_{N-1}<(k-1) l<$ $x_{N}<k l$, with the exception that if $N$ is odd $(N=2 k-1)$, then the middle knot $x_{k}$ 
$=0$ is the only knot that lies in the interval $(-l, l)$. It can also be shown that as $l \rightarrow \infty$, then $k l-x_{N}=k l-x_{N}(l) \rightarrow \infty$, since the error must remain below $\epsilon$. Hence, $s_{l}(t)-f_{l}(t) \longrightarrow 0$ for $t \in\left(x_{N}(l), k l\right)$, and we have

$$
\lim _{l \rightarrow \infty} \inf \left\|f_{l}-s_{l}\right\|_{2} \geqslant 2 \epsilon,
$$

where one $\epsilon$ is obtained from the error estimate on each of the intervals $[-k l-1$, $-k l]$ and $[k l, k l+1]$. Thus, there is a positive $l_{0}$, such that for each $l \geqslant l_{0}$,

$$
\left\|f_{l}-s_{l}^{*}\right\|_{2}<\left\|f_{l}-s_{l}\right\|_{2}
$$

contradicting that $s_{l}$ is the best $L_{2}$ approximant of $f_{l}$ from $S_{N}^{2}[-k l-1, k l+1]$. In particular, $f_{l_{0}}$ has more than one best $L_{2}$ approximant from $S_{N}^{2}\left[-k l_{0}-1, k l_{0}+1\right]$. By a standard smoothing technique, one can smooth the "corners" of $f_{l_{0}}$, and in fact, make $f_{l_{0}}$ strictly convex, without affecting the best approximants too much. Finally, by a simple translation, one can change the interval from $\left[-k l_{0}-1, k l_{0}+1\right]$ to $[0,1]$.

7. Application to $L_{1}$ Approximation. We now turn our attention to best $L_{1}[0,1]$ approximation of convex functions from $S_{N}^{2}$. In fact, we will show that Theorems 1,2 and 3 hold when $L_{2}$ is replaced by $L_{1}$. Furthermore, essentially the same results hold for a one-sided best $L_{1}$ approximation problem as indicated below.

We first note that Lemma 1 holds in the $L_{1}$ case if $(f-s)$ is replaced by $\operatorname{sgn}(f-s)$ and Lemma 2 is also true in $L_{1}$ [9]. If $f$ is a convex function on the interval $[\alpha, \beta]$ then the best linear $L_{1}[\alpha, \beta]$ approximant is obtained by interpolating $f$ at $\alpha+$ $(\beta-\alpha) / 4$ and $\alpha+3(\beta-\alpha) / 4$. In analogy with Eq. (3.2) we define the functional

$$
\begin{aligned}
F_{i}= & F_{i}\left(\mathrm{t}^{N}, f\right)=f\left(t_{i}+h_{i} / 4\right)-\left[f\left(t_{i}+3 h_{i} / 4\right)-f\left(t_{i}+h_{i} / 4\right)\right] / 2 \\
& -\left\{f\left(t_{i-1}+h_{i-1} / 4\right)+3\left[f\left(t_{i-1}+3 h_{i-1} / 4\right)-f\left(t_{i-1}+h_{i-1} / 4\right)\right] / 2\right\}
\end{aligned}
$$

Setting $\mathbf{F}=\mathbf{F}\left(\mathbf{t}^{N}, f\right)=\left(F_{1}, \ldots, F_{N}\right)$, we see that if $f$ is convex and $s\left(\mathbf{t}^{N} ; \cdot\right)$ is a best $L_{1}[0,1]$ approximant to $f$, then $\mathbf{F}\left(\mathbf{t}^{N}, f\right)=\mathbf{0}$.

One can now compute $J\left(\mathbf{F}\left(\mathbf{t}^{N}\right)\right)$ as in Section 3, yielding for instance

$$
\begin{aligned}
& \alpha_{i, i-1}=-h_{i-1}(3 / 8) \int_{1 / 4}^{3 / 4} f^{\prime \prime}\left(t_{i-1}+\tau h_{i-1}\right) d \tau, \quad 2 \leqslant i \leqslant N, \\
& \alpha_{i, i+1}=-h_{i}(3 / 8) \int_{1 / 4}^{3 / 4} f^{\prime \prime}\left(t_{i}+\tau h_{i}\right) d \tau, \quad 1 \leqslant i \leqslant N-1 .
\end{aligned}
$$

Furthermore, (7.1) can be rewritten in the form

$$
\begin{aligned}
F_{i}\left(\mathrm{t}^{N}\right)= & h_{i-1}^{2} \int_{0}^{1} \hat{w}(\tau) f^{\prime \prime}\left(t_{i}-\tau h_{i-1}\right) d \tau \\
& -h_{i}^{2} \int_{0}^{1} \hat{w}(\tau) f^{\prime \prime}\left(t_{i}+\tau h_{i-1}\right) d \tau,
\end{aligned}
$$

where $\hat{w}(\tau)=\tau-3 / 2(\tau-1 / 4)_{+}+1 / 2(\tau-3 /)_{+}$is a nonnegative weight function on $[0,1]$.

We are now in a position to state 
THEOREM 4. Let $f \in C^{2}[0,1]$ with $f^{\prime \prime}>0$ on $[0,1]$. If $\log f^{\prime \prime}$ is concave on $(0,1)$, then there exists a unique $s^{*} \in S_{N}^{2}$ which is a best $L_{1}[0,1]$ approximant to $f$.

THEOREM 5. Let $f \in C^{5}[0,1]$ with $f^{\prime \prime}>0$ on $[0,1]$. There exists a positive integer $N_{0}$ such that for every $N \geqslant N_{0}$, f has a unique best $L_{1}[0,1]$ approximant from $S_{N}^{2}$.

THEOREM 6. For any integer $N$ there exists a function $f \in C^{\infty}[0,1]$ with $f^{\prime \prime}>$ 0 on $[0,1]$ which does not have a unique best $L_{1}[0,1]$ approximant from $S_{N}^{2}$.

The proofs of Theorems 4, 5, and 6 closely parallel those of Theorems 1, 2, and 3 , respectively.

We next consider the following one-sided best $L_{1}[0,1]$ approximation problem from $S_{N}^{2}$. This problem can be treated as a problem of interpolation by functions from $S_{N}^{2}$ at the (variable) knots with minimum error. Let $f$ be a uniformly convex function on $[0,1]$. The problem is to study the uniqueness and eventual uniqueness of an $s^{*}(\cdot)=s^{*}\left(\mathrm{t}^{N}, \cdot\right)$ from $S_{N}^{2}$, with knot sequence $\mathrm{t}^{N}=\left(t_{1}^{*}, \ldots, t_{N}^{*}\right) \in \Sigma^{N}$, such that

$$
s^{*}\left(t_{i}^{*}\right)=f\left(t_{i}^{*}\right), \quad i=0, \ldots, N+1, \quad \text { and }
$$

$$
\left\|f-s^{*}\right\|_{1}=\inf \left\{\|f-s\|_{1}: s(\cdot)=s\left(\mathrm{t}^{N} ; \cdot\right) \in S_{N}^{2}, s\left(t_{i}\right)=f\left(t_{i}\right), 0 \leqslant i \leqslant N+1\right\}
$$

We have the following results.

THEOREM 7. Let $f \in C^{2}[0,1]$ with $f^{\prime \prime}>0$ on $[0,1]$. For $N=1$, there exists a unique $s^{*} \in S_{1}^{2}$ satisfying (7.5) and (7.6). For $N \geqslant 2$ if, in addition, $\log f^{\prime \prime}$ is concave on $(0,1)$, then there exists a unique $s^{*} \in S_{N}^{2}$ satisfying $(7.5)$ and (7.6).

THEOREM 8. Let $f \in C^{5}[0,1]$ with $f^{\prime \prime}>0$ on $[0,1]$. There exists a positive integer $N_{0}$ such that for every $N \geqslant N_{0}$, there is a unique $s^{*} \in S_{N}^{2}$ satisfying (7.5) and (7.6).

THEOREM 9. Let $N \geqslant 2$. There exists a function $f \in C^{\infty}[0,1]$ with $f^{\prime \prime}>0$ on $[0,1]$, such that the function $s^{*} \in S_{N}^{2}$ that satisfies (7.5) and (7.6) is not unique.

In proving Theorems 7 and 8 , we again derive the analogous quantities $F_{i}$, namely,

$$
\begin{aligned}
F_{i} & =f\left(t_{i+1}\right)-f\left(t_{i-1}\right)-f^{\prime}\left(t_{i}\right)\left(t_{i+1}-t_{i-1}\right) \\
& =h_{i}^{2} \int_{0}^{1} \widetilde{w}(t) f^{\prime \prime}\left(t_{i}+t h_{i}\right) d t-h_{i-1}^{2} \int_{0}^{1} \widetilde{w}(t) f^{\prime \prime}\left(t_{i}-t h_{i-1}\right) d t,
\end{aligned}
$$

where $\tilde{w}(t)=1-t$. These $F_{i}$ are obtained by differentiating the error functional with respect to the knots. Hence, for $f(t)=t^{2}, F_{i}=0, i=1, \ldots, N$, gives $t_{i}=i /(N+1)$, $i=1, \ldots, N$. Also, for each $i, 1 \leqslant i \leqslant N$, we obtain

$$
\sum_{j=1}^{N} \frac{\partial F_{i}}{\partial t_{j}}=h_{i}^{2} \int_{0}^{1} \tilde{w}(\tau) f^{\prime \prime \prime}\left(t_{i}+\tau h_{i}\right) d \tau-h_{i-1}^{2} \int_{0}^{1} w(\tau) f^{\prime \prime \prime}\left(t_{i}-\tau h_{i-1}\right) d \tau
$$

Therefore, by the same argument as in the $L_{2}$ case, we have Theorems 7 and 8 . To obtain Theorem 9, we just use the same function constructed in the proof of Theorem 3 and a slightly different proof.

Department of Mathematics

Texas A \& M University

College Station, Texas $\mathbf{7 7 8 4 3}$

1. D. L. BARROW, C. K. CHUI, P. W. SMITH \& J. D. WARD, "Unicity of best $L_{2}$ approximation by second-order splines with variable knots," Bull. Amer. Math. Soc., v. 83, 1977, pp. 1049-1050. 
2. C. DE BOOR, "Splines as linear combinations of B-splines. A survey," in Approximation Theory. II (G. G. Lorentz, C. K. Chui, L. L. Schumaker, Eds.), Academic Press, New York, 1976, pp. $1-47$.

3. C. DE BOOR, "On the approximation by $\gamma$ polynomials," in Approximation with Special Emphasis on Spline Functions (I. J. Schoenberg, Ed.), Academic Press, New York, 1969, pp. 157183.

4. D. BRAESS, “On the nonuniqueness of monosplines with least $L_{2}$-norm," J. Approximation Theory, v. 12, 1974, pp. 91-93.

5. C. K. CHUI, P. W. SMITH \& J. D. WARD, "On the smoothness of best $L_{2}$ approximants from nonlinear spline manifolds," Math. Comp., v. 37, 1977, pp. 17-23.

6. S. KARLIN, C. A. MICCHELLI, A. PINKUS \& I. J. SCHOENBERG, Studies in Spline Functions and Approximation Theory, Academic Press, New York, 1976.

7. G. MEINARDUS, Approximation of Functions: Theory and Numerical Methods, Translated by L. L. Schumaker, Springer-Verlag, New York, 1967.

8. J. T. SCHWARTZ, Nonlinear Functional Analysis, Courant Inst. of Math. Sci., New York, 1963.

9. P. W. SMITH, "On the smoothness of local best $L_{p}$ spline approximations," in Approximation Theory, II (G. G. Lorentz, C. K. Chui, L. L. Schumaker, Eds.), Academic Press, New York, 1976, pp. 563-566. 